\title{
Wilk, Martin (2020): Fragile kollektive Identitäten. Wie sich soziale Bewegungen radikalisieren
}

Bielefeld: transcript Verlag. 276 Seiten. $50 €$

\author{
Simon Bein $\mathbb{D}$
}

Angenommen: 21. Dezember 2020 / Online publiziert: 12. Januar 2021

(C) Der/die Autor(en) 2021

Die Proteste gegen Rassismus und Diskriminierung in den USA erreichten im Sommer 2020 bekanntlich einen neuerlichen Höhepunkt. Der zentrale Akteur dahinter, die Black-Lives-Matter-Bewegung, hat auch über die Vereinigten Staaten hinaus an Einfluss und Mobilisierungspotenzial gewonnen. Dabei geht es in den Protesten nicht nur um die konkrete Kritik an Polizeieinsätzen und an Vorfällen alltäglicher Diskriminierung, sondern um die Anerkennung einer ganzen Gesellschaftsgruppe. Dass dieser Identitätskonflikt unter der Präsidentschaft Trumps in einer gewissen Radikalität zum Vorschein kommt, scheint kein Zufall zu sein, denn auf der anderen Seite steht der Versuch von Trump und seinen Anhängern, die ursprüngliche White Identity der Mehrheitsgesellschaft zu bewahren. Identitätspolitik, wie dieses Beispiel zeigt, ist dabei nicht zuletzt seit Francis Fukuyamas viel diskutierter Monografie über die Bedeutung von Anerkennungskämpfen und Identitätspolitiken zum zentralen Paradigma der Erklärung sozialer und politischer Polarisierung geworden.

Die vorliegende Studie des Kulturwissenschaftlers Martin Wilk könnte also kaum aktueller sein, wenn sie fragt, warum sich soziale Bewegungen radikalisieren und welche Rolle Identitätskonstruktionen dabei spielen. Um einen gewissen „Abstand zu den häufig aufgeregten tagespolitischen Debatten“ (S. 11) zu gewinnen, wählt der Autor ein Beispiel der Bürgerrechtsbewegung der 1960er-Jahre als Fallstudie. Das Student Nonviolent Coordinating Committee (SNCC) symbolisiere dabei eine Protestbewegung, deren Entwicklung von ihrer Gründung 1960 bis zur Auflösung 1969 ,von einem absolut pazifistischen zu einem gewaltbefürwortenden Protest“ (S. 73) mit paramilitärischem Anstrich verlaufen ist und insofern in ihrer Radikalisierungsdynamik einen außergewöhnlichen Fall darstellt.

S. Bein $(\triangle)$

Institut für Politikwissenschaft, Universität Regensburg, Regensburg, Deutschland

E-Mail: Simon.Bein@politik.uni-regensburg.de 
Aber dient das Konzept kollektiver Identität wirklich zur Erklärung von radikalem Handeln in Protestbewegungen? Ja, so die zentrale These Wilks in Anknüpfung an den schwedischen Politikwissenschaftler Erik Ringmar: Radikalisierung vollzieht sich als Antwort auf eine Bedrohungsperzeption der eigenen Identität, „wenn alte Identitätsbezüge durch politischen, ökonomischen und sozialen Wandel in Auflösung begriffen sind, sprich die Narrationen über das eigene Selbstbild prekär werden“ (S. 70). Dabei grenzt Wilk seinen Ansatz im zweiten Kapitel explizit von gängigen Theorien der Bewegungsforschung ab, die Radikalisierung durch mangelnden Erfolg oder verwehrten Zugang zu Ressourcen sowie politische Gelegenheitsstrukturen zu erklären versuchen. Der SNCC hingegen radikalisierte sich in einer Phase, in der durchaus Erfolge der Bürgerrechtsbewegung zu erkennen waren, sich die soziale und ökonomische Lage vieler Schwarzer verbesserte und die Bewegung durchaus über politische Einflussmöglichkeiten verfügte. Die zentrale Frage von Wilks Monografie lautet also anders ausgedrückt: „Warum radikalisieren sich politische Akteure, obwohl ihnen auch andere Handlungsalternativen offenstehen“ (S. 17)?

In Erweiterung der bisherigen Angebote der Radikalisierungsforschung erfolgen im dritten Kapitel die Einführung des Konzeptes der kollektiven Identität und die Synthese mit den Annahmen zur Radikalisierung von sozialen Bewegungen. Angesichts der gegenwärtigen Bedeutung von Identitätstheorien und der gleichzeitig notorischen Unschärfe des Identitätsbegriffes erscheint dieser Abschnitt allerdings etwas knapp, soll doch hier das ,,analytische Werkzeug“ (S. 64) eingeführt werden: In Anbindung an eine Definition kollektiver Identität von Alberto Melucci sei es das Ziel der Studie, identitätsstiftende Narrative auf der kognitiven, relationalen und emotionalen Ebene sowie deren Wandel zu analysieren. Die Antwort darauf liefert eine diskursanalytische Aufbereitung der internen Identitätskonstruktion des SNCC, für die Wilk auf umfangreiches empirisches Material aus dem Inneren der Organisation zurückgreift. Die Ausarbeitung der „organisationsinternen Diskurse“ (S. 19) ist daher der zentrale empirische Kern der Monografie in den Abschnitten fünf bis sieben, nachdem in Kapitel vier die bisherigen Theorien der Radikalisierung des SNCC vorgestellt werden.

Der empirische Teil spiegelt dabei in seinem Aufbau drei Phasen der Radikalisierung der Organisation wider: Erstens von der Gründung 1960 bis zum Juni 1962, wo friedliche Proteste und Aktionen gegen die Segregation in den Südstaaten im Mittelpunkt standen; zweitens bis zum Inkrafttreten des Voting Rights Acts 1965, primär von Projekten zur Wählerregistrierung begleitet; und drittens bis zum Jahr 1968, wo sich eine Form des ,black nationalism“ gepaart mit separatistischem Aktivismus abzeichnete und die Isolation der Gruppe vorantrieb. Im Verlauf dieser Phasen zeigt Wilk schließlich überzeugend, wie sich zunächst identitätsstiftende Narrative (Integration in die Mehrheitsgesellschaft, Wählerregistrierung, politische Teilhabe) durch Erfolge und sich verändernde Rahmenbedingungen auflösten und eine zusehends exklusive Identitätspolitik hervorbrachten.

Die Monografie zeigt insgesamt sehr aufschlussreich und theoretisch fundiert, wie und warum Radikalisierung von kollektiven Akteuren als Antwort auf Identitätsfragen auftreten kann. Wilk plädiert in nachvollziehbarer Weise dafür, Prozesse der Konstruktion kollektiver Identität in radikalen Bewegungen stärker zu berücksichtigen. Denkt man einige Argumente der Studie weiter, erscheint es auch mit Blick 
auf die Radikalisierungsdebatten der Gegenwart - die Querdenken-Bewegung oder PEGIDA in Deutschland, aber auch die Black-Lives-Matter-Bewegung in den USA sind als Beispiele zu nennen - angezeigt, deren Proteste nicht als nur als Akte individualpsychologischer Verzweiflung und Irrationalität abzutun, sondern die veränderte Logik der Rationalität in den Blick zu nehmen. Denn, so Wilk, die Radikalisierung von Identitätspolitik stelle keinesfalls automatisch die Aufgabe jeglicher Rationalität dar, sondern könne auch als eine reflektierte Strategie zur Stabilisierung kollektiver Identität interpretiert werden, indem die innere Kohäsion und Abgrenzung nach außen gestärkt wird.

Als einzige Schwäche ist zu nennen, dass die doch sehr zahlreichen theoretischen Einzelbestandteile zur besseren Nachvollziehbarkeit in ein übersichtliches Gesamtkonzept eingebettet hätten werden können, anstatt nacheinander und stark verschachtelt eingeführt zu werden. So werden beispielsweise an den zentralen Stellen des Theorieteils in direkter Abfolge drei Dimensionen kollektiver Identität unterschieden, der analytische Rahmen um die Differenzierung in Makro-, Meso- und Mikroebene erweitert und schließlich das Konzept der Narration als leitender Ansatz eingeführt (S. 64-72). Die an einigen Stellen daher wenig synthetisch erscheinende Komplexität des theoretischen Ansatzes soll jedoch keinesfalls das Verdienst der Studie Wilks schmälern, die eine innovative Verbindung des Konzeptes kollektiver Identität mit der Radikalisierungs- und Bewegungsforschung leistet und daher auch über die Kulturwissenschaft hinaus einen Beitrag zu den gegenwärtigen Debatten zu Radikalisierung und Identitätspolitik bietet.

Funding Open Access funding enabled and organized by Projekt DEAL.

Open Access Dieser Artikel wird unter der Creative Commons Namensnennung 4.0 International Lizenz veröffentlicht, welche die Nutzung, Vervielfältigung, Bearbeitung, Verbreitung und Wiedergabe in jeglichem Medium und Format erlaubt, sofern Sie den/die ursprünglichen Autor(en) und die Quelle ordnungsgemäß nennen, einen Link zur Creative Commons Lizenz beifügen und angeben, ob Änderungen vorgenommen wurden.

Die in diesem Artikel enthaltenen Bilder und sonstiges Drittmaterial unterliegen ebenfalls der genannten Creative Commons Lizenz, sofern sich aus der Abbildungslegende nichts anderes ergibt. Sofern das betreffende Material nicht unter der genannten Creative Commons Lizenz steht und die betreffende Handlung nicht nach gesetzlichen Vorschriften erlaubt ist, ist für die oben aufgeführten Weiterverwendungen des Materials die Einwilligung des jeweiligen Rechteinhabers einzuholen.

Weitere Details zur Lizenz entnehmen Sie bitte der Lizenzinformation auf http://creativecommons.org/ licenses/by/4.0/deed.de. 